months decreased mean BMD by $2,6-3,5 \%$ (depending on the BMD site) compared to mean BMD decreases of $1,9-2,9 \%$ with $\mathrm{LMWH}$. BMD of the spine and hip is significantly lower in patients with LMWH therapy than in subjects with UFH therapy, which is independent of age, sex, menopause, low BMI and altered body composition.

Conclusions: LMWH most likely have less effect on bone turnover when compared to UFH. LMWH for 6 months may not increase the risk of osteoporosis, but longer exposure for up to 24 months may adversely affect BMD. Clinicians should consider monitoring BMD in adults on long-term heparin therapy who are at increased risk of bone loss or fracture.

Disclosure of Interest: None declared

DOI: 10.1136/annrheumdis-2018-eular.4255

\section{THU0501 WHAT DOES TRABECULAR BONE SCORE CONTRIBUTE TO A FRACTURE LIAISON SERVICE?}

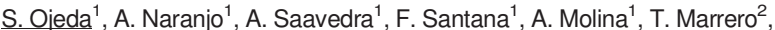
O. Suarez ${ }^{2}$, C. Rodriguez-Lozano ${ }^{1} .{ }^{1}$ Rheumatology, ${ }^{2}$ Radiology, Hospital Univ. Gran Canaria Dr. Negrin, Las Palmas de Gran Canaria, Spain

Background: The utility of the trabecular bone score (TBS) is controversial. Objectives: To analyse the clinical usefulness of FRAX-TBS in patients with fragility fracture seen in a Fracture Liaison Service (FLS) compared to FRAX without TBS.

Methods: Consecutive outpatients $>50$ y seen in our FLS were included, excluding patients admitted with hip fracture. The variables included were: age, sex, type of fracture, risk factors for FRAX, FRAX for major and hip fractures with and without TBS, bone densitometry (DXA) and indication of treatment to prevent new fractures.

Results: We included 251 patients, mean age 69 y, $86 \%$ women. The most frequent type of fracture was the forearm $(n=122)$ followed by the humerus $(n=64)$. The DXA scan results (taking the lowest value of column/hip) was osteoporosis in $41 \%$. The average TBS was 1.307 (SD 0.103 , range $0.961-1.550$ ): forearm fracture 1.313 (SD 0.102), vertebra 1.281 (SD 0.131) and hip 1.291 (SD 0.103). 32\% presented normal TBS result $53 \%$ partially degraded and $15 \%$ degraded (table 1 ). The results of FRAX with and without TBS were similar, with an average for major/ hip fracture of 10.7/4.2 with DXA and 10.6/4.0 with TBS respectively ( $p>0.05)$. A high risk of hip fracture (FRAX $\geq 3$ ) was observed in $43 \%$ of patients using FRAXDXA and $41 \%$ using FRAX-TBS. Ans a high risk of major fracture (FRAX $\geq 10)$ in $40 \%$ of patients using FRAX-DXA and $41 \%$ using FRAX-TBS. In 241 cases (96\%) there was concordance in level of risk for FRAX-DXA and FRAX-TBS, while the 10 discordant cases for high risk of fracture were distributed as follows: in 6 cases FRAX-DXA was high and FRAX-TBS was normal, and in 4 cases FRAX-DXA was normal and FRAX-TBS was elevated. In our FLS, a bisphosphonate was prescribed to 169 patients $(67 \%)$. Regarding patients with a low FRAX risk $(n=82)$, in one patient FRAX-TBS was in favour of treatment. Thus, in 1/251 fractures FRAXTBS could have influenced the treatment indication compared to FRA-DXA

\begin{tabular}{lccc}
\hline & $\begin{array}{c}\text { TBS } \geq \mathbf{1 . 3 5 0} \\
\text { Normal }\end{array}$ & $\begin{array}{c}\text { TBS }<\mathbf{1 . 3 5 0} \mathbf{y} \geq \mathbf{1 . 2 0 0} \\
\text { partially degraded }\end{array}$ & $\begin{array}{c}\text { TBS }<1.200 \\
\text { Degraded }\end{array}$ \\
\hline Number of patients & 80 & 134 & 37 \\
Age, mean (SD) & $67(11)$ & $70(10)$ & $71(10)$ \\
Sex, women, $\mathrm{n}(\%)$ & $65(81)$ & $124(92)$ & $33(92)$ \\
Risk factors, $\mathbf{n}(\%)$ & & & \\
BMl, mean & 27 & 29 & 31 \\
Previous fracture & $8(10)$ & $34(25)$ & $7(19)$ \\
Parent's hip fracture & $8(10)$ & $21\left({ }^{16}\right.$ & $2(5)$ \\
Glucocorticoids & $6(8)$ & $5(4)$ & $5(13)$ \\
Smoking & $9(11)$ & $20(15)$ & $6(16)$ \\
Alcohol & $2(3)$ & $4(3)$ & $1(3)$ \\
Rheumatoid arthritis & $3(4)$ & $2(1)$ & 0 \\
Secondary osteoporosis & $13(16)$ & $22(18)$ & $10(28)$ \\
Bone Densitometry, $\mathbf{n}(\%)$ & & & \\
Normal & $24(30)$ & $7(5)$ & $3(8)$ \\
Osteopenia & $37(46)$ & $65(49)$ & $12(32)$ \\
Osteoporosis & $19(24)$ & $62(46)$ & $22(60)$ \\
\hline
\end{tabular}

Conclusions: In our FLS unit, TBS does not help to classify patients' risk. The values of FRAX-TBS are similar to those of FRAX-DXA, not offering advantages when classifying patients with high risk who are candidates for treatment.

Disclosure of Interest: None declared

DOI: 10.1136/annrheumdis-2018-eular.2722

\section{THU0502 \\ ASSESSMENT OF SEVERITY OF ATHEROSCLEROTIC LESIONS OF CAROTID ARTERIES IN MEN WITH CORONARY HEART DISEASE DEPENDING ON BONE MINERAL DENSITY AND RISK OF OSTEOPOROTIC FRACTURES}

T. Raskina ${ }^{1}$, A. Voronkina ${ }^{2}$, M. Letaeva ${ }^{1}$, A. Kokov ${ }^{3}$, O. Barbarash ${ }^{3}$, E. Maluta ${ }^{2}$ ${ }^{1}$ Federal State Educational Institution of Higher Education "Kemerovo State Medical University"; ${ }^{2}$ State Autonomous Healthcare Institution Kemerovo region "Regional Clinical Hospital of Emergency Medical Care named M. Podgorbunsky", ${ }^{3}$ Federal State Budgetary Scientific Institution "Scientific-Research Institute of Complex Issues of Cardiovascular Disease", Kemerovo, Russian Federation

Objectives: To assess severity of atherosclerotic lesions of carotid arteries (CA) in men with coronary heart disease (CHD) depending on bone mineral density (BMD) and risk of osteoporotic fractures according to FRAX scale.

Methods: The study involved 102 men aged $51-75$ years (median age of $61^{55 ; 65}$ with $\mathrm{CHD}$, verified by coronary angiography method. All patients underwent dua energy X-ray absorptiometry of lumbar vertebral bodies LI-LIV and femoral neck and colour duplex scanning of extracranial arteries. Assessment of severity of carotid atherosclerosis was carried out by presence of atherosclerotic plaques (ASP) and stenosis of CA, thickness of intima-media (TIM) CA. Threshold TIM for men over age of 50 was considered $0.9 \mathrm{~mm}$ (recommendations of American Society of Echocardiography, 2008 Based on results of densitometry value of T-criterion (recommendations ISCD, 2007 men were divided into three groups: 33 patients with osteoporosis (OP, T-criterion $\leq 2.5$ ), 48 patients with osteopenia (OPE, T-criterion of -1 to -2.5 ) and 21 men with normal BMD (NBMD, T-criterion $\geq-1$ ). On the basis of information on the presence of clinical risk factors of osteoporotic fractures and densitometry data at all included in the study patients FRAX calculato was used to quantify probability of major osteoporotic fractures and hip fracture in next 10 years.

Results: It was found that $86.3 \%$ of patients value TIM exceeds threshold value $(0.9 \mathrm{~mm})$. Thickening of intima-media complex was in men with OP in $88.0 \%$ of cases, with OPE - in $87.5 \%$, with NBMD - in $81.0 \%$ without statistically significant differences $(p>0.05)$. In a comparative analysis of atherosclerotic lesions CA found that men with OP significantly were more likely to have ASP in CA $(75.8 \%)$ compared to patients with OPE $(43.7 \%, p=0.010)$ and NBMD $(38.1 \%, p=0.016)$. Stenosis of CA in all investigated men was discovered in 54 cases (53\%). Comparative analysis showed that in patients with OP percentage of patients with stenosis of CA was 2 times more than men with NBMD $(75.8 \%$ vs $38.1 \%, p=0.006)$ and was significantly higher than proportion of such patients in the group with OPE $(44.0 \%, p=0.004)$. In all included the study patients 10 year absolute risk of major osteoporotic fracture by FRAX was equal to $9.88 \pm 7.22$, risk of hip fracture -3.97 \pm 6.27 . There was inverse correlation of TIM with $T$-criterion $(r=-0.21, p=0.035)$ and with BMD ( $r=-0.20, p=0.045)$ at level of hip and reliable direct correlation between TIM and risk of hip fracture by FRAX ( $r=0.21, p=0.035)$.

Conclusions: In men with CHD aged over 50 years low BMD (T-criterion is less than 2.5) is associated with more severe carotid atherosclerosis, and thickening of intima-media CA - with an increased risk of hip fracture.

Disclosure of Interest: None declared

DOI: 10.1136/annrheumdis-2018-eular.5548

\section{THU0503 COST ANALYSIS OF ANTI-OSTEOPOROTIC DRUGS IN REAL-WORLD CLINICAL PRACTICE}

V. Orlando ${ }^{1}$, V.M. Monetti ${ }^{1}$, F. Guerriero ${ }^{1}$, V. Russo ${ }^{1}$, R. Piscitelli ${ }^{1}$, M. Ruggeri ${ }^{2}$, E. menditto ${ }^{1} .{ }^{1}$ CIRFF Center of Pharmacoeconomics, University of Naples Federico II, Naples; ${ }^{2}$ School of Economics, Catholic University of Sacred Heart, Rome, Italy

Background: Nonadherence can reduce treatment effectiveness and can negatively affect healthcare costs and thus the treatments' cost-effectiveness. Adherence in the setting of osteoporosis has been shown to be just as problematic, if not worse, than that in other chronic diseases. Economic evaluations based on modelling are commonly used to compare alternative treatment strategies in osteoporosis, to support decision-makers and to inform treatment guidelines.

Objectives: The aim of this study is to analyse healthcare costs of osteoporosis and to build a economic model cost-effectiveness of pharmacological intervents based on real world data.

Methods: The data of the analysed subjects were drawn from the administrative databases of four Local Health Authorities in the Abruzzo Region. Patients 60 years of age or older were included if at least one prescription for any 
antiosteoporosis drugs had been filled in between January 1, 2006 and December 31,2006 . The patients were classified as exposed or not exposed to osteoporotic fracture. For each group, compliance with antiosteoporosis drugs was calculated. Patients were considered compliant if their Medication Possession Ratio (MPR) was $\geq 80 \%$. The cost analysis was conducted taking each healthcare service into account, i.e. drug therapy, diagnostic tests and hospitalisation admissions, during the study period. A hypothetical scenario based on the real-life available evidence was constructed. The mean level of adherence to populate the hypothetical scenario of "full adherence" was set at MPR $>80 \%$. The model built by adding a step value, constrained by a normal random variable, to the real-word adherence of each subject so that the subject shifted to the hypotetical scenario of full adherence, in order to quantify the clinical outcome (number of fractures) achievable in the hypotetical scenario. Cost-effectiveness of full adherence compared to real world adherence was expressed in terms of Incremental Cost Effectiveness Ratio (ICER) and the number of fractures avoided was set as an effectiveness unit of measure.

Results: The mean annual healthcare cost per fracture avoided was $€ 247.44$, of which medical treatments and diagnostic tests accounted for $€ 103.60$ (41.9\%) and $€ 143.84(58.1 \%)$, respectively. The mean annual helathcare cost per fractured patient was $€ 1,044.85$, of which medical treatments, diagnostic tests and hospitalizations for osteoporotic fracture accounted for $€ 88.73$ (8.5\%), $€ 169.48$ $(16.2 \%)$ and $€ 786.65$ (75.3\%), respectively.

Conclusions: Costs per fractured patients resulted to be about four times greater than those of not fractured patients. Therefore, only enhancing adherence to medication may lead to reductions in the number of patients requiring hospitalisation.

\section{REFERENCES:}

[1] Hiligsmann M, Boonen A, Rabenda V, Reginster JY. The importance of integrating medication adherence into pharmacoeconomic analyses: the example of osteoporosis 2012;159-166.

[2] Scotti L, Arfè A, Zambon A, Merlino L, Corrao G. Cost-effectiveness of enhancing adherence with oral bisphosphonates treatment in osteoporotic women: an empirical approach based on healthcare utilisation databases. BMJ open 2014;4(3),e003758.

Disclosure of Interest: None declared

DOI: 10.1136/annrheumdis-2018-eular.6620

\section{THU0504 UTILISATION OF ANTI-OSTEOPOROTIC DRUGS IN REAL-WORLD DATA: A STUDY OF GENDER-RELATED DIFFERENCES}

V. Orlando ${ }^{1}$, V.M. Monetti ${ }^{1}$, F. Guerriero ${ }^{1}$, V. Russo ${ }^{1}$, A. Moretti $^{2}$, A. Piscitelli $^{1}$ R. Piscitelli ${ }^{1}$, G. Iolascon ${ }^{2}$, E. Menditto ${ }^{1}$. ${ }^{1}$ CIRFF Center of Pharmacoeconomics, University of Naples Federico II; ${ }^{2}$ Department of Medical and Surgical Specialties and dentistry, University of Campania Luigi Vanvitelli, Naples, Italy

Background: Osteoporosis is mostly defined as the disease of women, because the prevalence and fracture rates are much higher in postmenopausal women than in older men. However, there has been increasing recognition that male osteoporosis also represents an important burden as a common cause of morbidity, mortality and health care expenditure. Also, men are more likely than women to have osteoporosis that is undiagnosed and undertreated.

Moreover, bone fracture are important factors of high mortality and morbidity rates in osteoporotic patients. Lack of persistence is common among subjects using oral anti-osteoporotic drugs, and leads to increased risk of fragility fracture.

Objectives: The aim of this study is to perform gender specific analysis regarding the persistence to antiosteoporosis drugs.

Methods: We conducted a retrospective cohort study using administrative data from four local health authorities in the Abruzzo Region (Central Italy), which comprise about 900000 inhabitants (68\% of the overall regional population). Patients 60 years of age or older were included if at least one prescription for any antiosteoporosis drugs had been filled in between January 1, 2006 and December 31, 2006. Persistence estimates over time (discontinuation rates were assessed at 365 days) were derived using Kaplan-Meier survival analysis, stratifying for gender, considering treatment discontinuation as failure event and comparing differences using Log-rank test (1 degree of freedom).

Results: The final cohort consisted of a total of 7867 patients ( $87.2 \%$ women). The mean patient age for both genders at the index date was 74.5 years. The crude analysis of long-term gender persistence showed a significant difference between women and men: the relative number of persistence patients after 1 year was $66.4 \%$ in men and $44.7 \%$ in women. The Kaplan Meier plots of time to persistence start to differ for men vs women approximately 60 days after treatment start (figure 1).

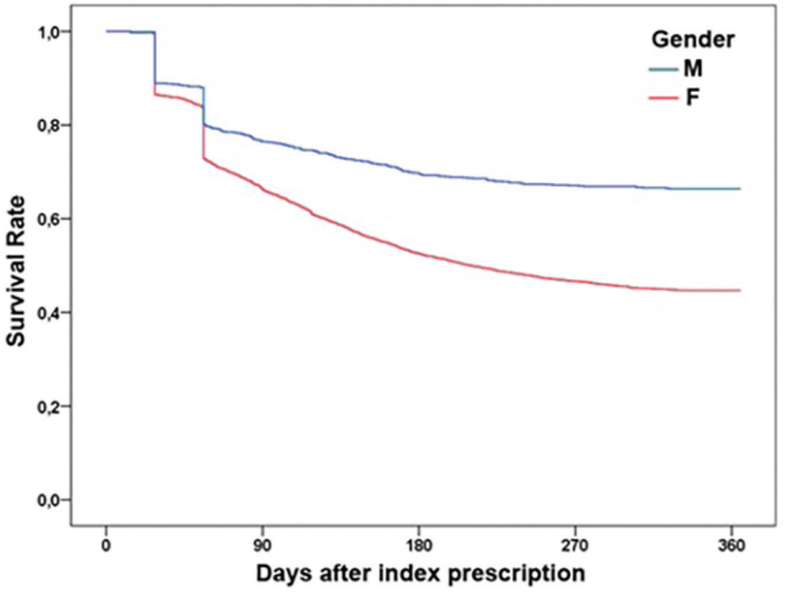

Conclusions: The results showed that gender influences adherence to therapy and this is an issue that could be taken in strong consideration in tailor intervention to improve adherence.

\section{REFERENCES:}

[1] Cawthon PM. Gender differences in osteoporosis and fractures. Clinica Orthopaedics and Related Research 2011;469(7):1900-1905.

[2] Bor A, Matuz M, Gyimesi N, et al. Gender inequalities in the treatment of osteoporosis. Maturitas 2015;80:162-169.

[3] Christopher J, et al. Sex and gender considerations in male patients with osteoporosis. Clinical Orthopaedics and Related Research 2011;469 (7):1906-1912.

Disclosure of Interest: None declared

DOI: 10.1136/annrheumdis-2018-eular.5572

\section{THU0505 INFLUENCE OF ORAL PREDNISOLONE ON EFFECT OF DENOSUMAB ON OSTEOPOROSIS IN PATIENTS WITH JAPANESE RHEUMATOID ARTHRITIS; 36 MONTHS OF FOLLOW-UP A MULTICENTER REGISTRY STUDY}

Y. Kanayama ${ }^{1}$, Y. Hirano ${ }^{2}$, N. Takahashi ${ }^{3}$, S. Asai ${ }^{3}$, N. Ishiguro ${ }^{3}$, T. Kojima ${ }^{3}$, on behalf of TBCR-BONE study group. ${ }^{1}$ Orthopedic Surgery And Rheumatology, Toyota Kosei Hospital, Toyota; ${ }^{2}$ Rheumatology, Toyohashi Municipal Hospital, Toyohashi; ${ }^{3}$ Orthopedic Surgery, Nagoya University Graduate School of Medicine, Nagoya, Japan

Background: Denosumab (DMB) is a fully human monoclonal antibody to the receptor activator of nuclear factor-kappaB ligand (RANKL) that blocks its binding RANK, inhibiting the development and activity of osteoclasts, decreasing bone resorption, increasing bone density and reducing fracture risk. Osteoporosis(OP is more frequent in patients with rheumatoid arthritis(RA) than in the general population due to active systemic inflammation as well as the use of glucocorticoid and immobility. We investigated the influence of oral prednisolone on effect of DMB in patients with Japanese RA from initiation to 36 months at this time.

Objectives: This prospective study investigated the efficacy of DMB for 36 months on glucocorticoid- induced OP in RA patients.

Methods: Patients with a diagnosis of RA according to the 2010 ACR/EULAR criteria who had been prescribed DMB from Tsurumai Biologics Communication Registry (TBCR)-BONE between October 2013 and October 2014 were enrolled The final study cohort of 56 patients received continuous DMB therapy more than 36 months. The DMB dose was $60 \mathrm{mg}$ at once every 6 months. In all cases native or activated vitamin D has been used. We reviewed the results for 36 months about the increase and decrease of bone mineral density(BMD) of lumbar spine (LS) and total hip(TH) by DEXA and bone turnover markers, intact n-terminal propeptide type I procollagen(PINP) and tartrate-resistant acid phopshatate form $5 b$ (TRACP-5b).

Results: In the patients receiving oral prednisolone group $(\mathrm{n}=20, \mathrm{GC}+)$ and not receiving group $(n=36, G C-)$, the number of female was each $18(90 \%)$ and 35 $(98 \%)$ cases $(p=0.288)$. The mean age was $69.5 \pm 7.0$ and $70.5 \pm 6.6$ years old $(p=0.700)$; disease duration was $15.9 \pm 9.5$ and $16.3 \pm 13.7$ years $(p=0.688)$; the body mass index was $20.6 \pm 3.5$ and $19.5 \pm 3.0(p=0.225)$ and the FRAX was 33.8 